\title{
Use of Activation Values and Weight Space by Activation and Synaptic Dynamics for Network Reliability Measurement
}

\author{
Anuradha \\ Assistant Professor \\ MIET, Meerut
}

\author{
Harish Kumar \\ Associate Professor (CSE) \\ MIET, Meerut
}

\author{
Rakesh Kumar Arora \\ Associate Professor \\ KEC, Ghaziabad
}

\begin{abstract}
The problem which is occurring nowadays for networks is reliability as the networks are becoming more wide and complex. Reliability is the ability of a network to carry out a desired operation such as "communication". It is one of the most important aspects of networks for solving complex problems. The network should have minimum failure and should be cost-effective. The solution can be attained by using artificial neural network. An Artificial Neural Network is a highly simplified model of the structure of the biological neural network. It consists of interconnected processing units. The weight present between the connections is used for activation value. The inputs or outputs of the artificial neural networks could be discrete or continuous and also could be deterministic or stochastic. The activation dynamics determine the activation values of nodes. Synaptic dynamics is to adjust the weights in order to store given patterns in the network. This paper presents different measures of the reliability of the network by using Artificial Neural Network. The evaluation of the network is been done by studying various approaches to reliability measurement. The aim of the work is to present an optimized neural network approach to improve reliability with a high learning rate.
\end{abstract}

\section{Keywords}

ANN, Neuron, Activation Value, Network Reliability.

\section{INTRODUCTION}

Reliability is defined as the probability that each and every network node communicate with every remaining node in the network and it is practically very difficult to calculate exact reliability is growing variable-sized highly increasing networks from very small to large network size. Reliability measurement or calculation is an important aspect and components for solving many complex real-world problems which requires optimality in design so that cost and fault will be minimized [3]. The problem of finding exact reliability in highly increasing variable-sized growing networks is the NPHard problem [8] because the computational effort required to measure the exact reliability is growing exponentially with an increase in the number of nodes and links in the network. The optimal network design problem from a highly increasing variable-sized growing network is difficult and equal to NPHard, when exact reliability of each and every network node and link is considered [8]. Since reliability is defined as the probability that each and every network node communicate with every remaining node in the network and it is practically very difficult to calculate exact reliability is growing variablesized highly increasing networks from very small to large network size. The rest of the paper is organized into five sections. An overview of ANN is defined in Section III. The
Literature Review is given in Section II. The Reliability measuring Approach is explained in Section VII. Section VIII and IX defines Results and Conclusions. The Torus network is used for calculation of results. The example of graph is given in figure:

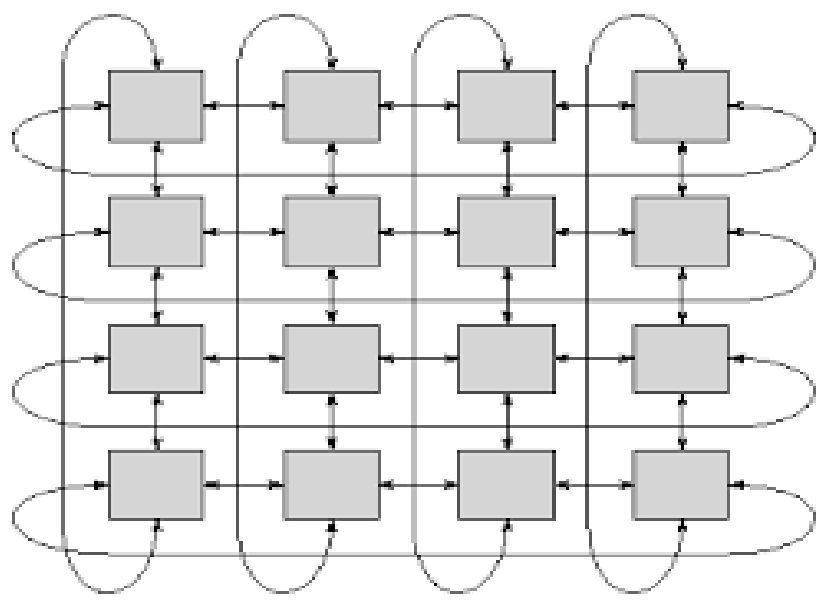

Figure 1: A Torus Network

\section{LITERATURE SURVEY}

Elperin [4] considers the renewal of network parameters when the failure occurs. The mean time for failure and operational components are important in calculating the overall reliability. But the proposed method fails when the network is relatively complex

Shpungin [6] applied combinatorial approaches to Monte Carlo estimation for evaluating network reliability of dynamic systems parameters. Their proposal considers two types of dynamic networks for estimating the lifetime distributions of network reliability. The first system is a network with edges existing in time, which may be erased independently of each other. The second type of architecture is a network with renewable edges. Shpungin proposed a combinatorial approach for Monte Carlo reliability estimation of a network with unreliable nodes and unreliable edges.

Abdullah Konak and Alice E. Smith [3] proposed that highspeed networks have proliferated across the globe; their topologies have become sparser due to the increased capacity of communication media and cost considerations. Reliability has been a traditional goal within network design optimization of sparse networks. They propose a genetic approach that uses network resilience as a design criterion in order to ensure the integrity of network services in the event of component failures. They analyze the effect of noise in the simulation 
estimator used to evaluate network resilience on the performance of the proposed optimization approach.

Ramnathan [7] stated that the number n1of i-edge network cut-sets in a graph is an important parameter in reliability analysis. They had proved that number n1of i-edge network cut-sets can be determined in polynomial time based on the literature. They had given a polynomial-time algorithm for determining $\mathrm{r}+\mathrm{k}$ for any fixed $\mathrm{k}$ as a special case of counting cuts of specified maximum weight in an edge-weighted graph.

Z. Hongshi [9] stated the reliability of distributed systems \& computer networks in which computing nodes or communication links fail with certain probabilities which can be modeled by the probabilistic network. The proposed residual connectedness reliability (RCR) of the probabilistic network under the fault model in which both node and link faults may be useful but finding is NP-hard. Their approach suggests an improvement over lower and upper bounds which may tend to become zero or near to zero in large or smaller networks. Their method suggests an improvement in bounds by finding near-optimal subsets of nodes and links, but, incurs significant computational effort.

\section{ARTIFICIAL NEURAL NETWORK}

An Artificial Neural Network is a collection of the number of artificial interconnected units/neurons/nodes. Each node implements a local computation or function. The nodes are connected by the connection link. The connection link is associated with weights [1].

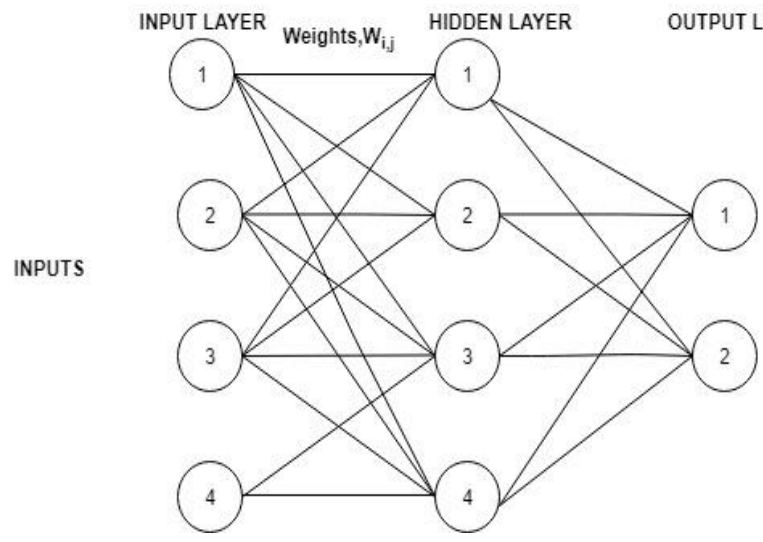

(a)
The I/O the characteristic can identify the output of any node because of the interconnection to the other nodes. The actual output can be determined by using the activation value at the output unit [2][3]. For the proper functioning of the artificial neural network, the rules governing the changes of the connection weight and activation values are also specified. The desired task is achieved by implying or specifying the rules in the activation and synaptic dynamics equations.

Activation dynamics identifies the activation values or activation state of all the nodes of the network as a function of time and also the dynamics of the output state of a network. The short term memory function is determined by activation states of a network [5][6].

The Activation dynamics recall or recollect the patterns which are stored in the network. Synaptic dynamics identify the weight vector as a function of time when the change in weights happens. The first derivative of the activation value of a neuron is defined as Activation dynamics. The given equation defines the activation function for $\mathrm{i}_{\mathrm{th}}$ neuron.

$$
\begin{aligned}
& \mathrm{x}_{\mathrm{i}}=\mathrm{d} \mathrm{x}_{\mathrm{i}} / \mathrm{dt}=\mathrm{h}(.) \\
& \mathrm{h}(.)=\text { Activation State and Synaptic weights }
\end{aligned}
$$

The below figure(b) is of $\mathrm{N}$ units. The potential of the cell membrane is generally allied with activation value

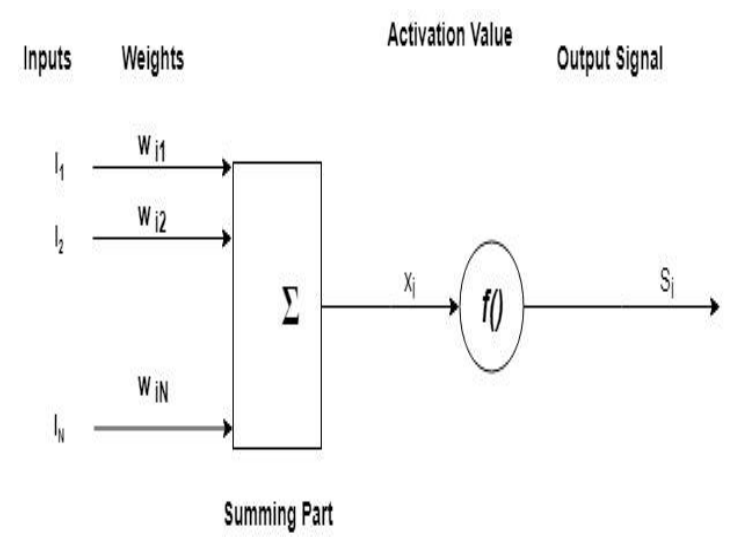

(b)

Figure 2: (a) Artificial Neural Network (b) Model of Artificial Neuron

The output produced at the axon due to given membrane potential is the output $\mathrm{f}($.). The activation function which is increasing function bounds the output.

\subsection{Activation Dynamics}

The first derivative expression of the activation value is been used for defining activation models. The cell membrane potential is inferred as activation value $\left(\mathrm{x}_{\mathrm{i}}\right)$.

$$
\mathrm{x}_{\mathrm{i}}=\mathrm{x}_{\mathrm{i}}(\mathrm{t})
$$

where $x_{i}(t)$ is rate of change of activation value.If an external excitatory input Ii is there and assumes resting potential to be zero then the additive activation model is given by

$$
\mathrm{x}_{\mathrm{i}}(\mathrm{t})=-\mathrm{T}_{\mathrm{i}} \mathrm{x}_{\mathrm{i}}(\mathrm{t})+\mathrm{U}_{\mathrm{i}} \mathrm{I}_{\mathrm{i}}
$$

where $U_{i}$ is the constant that is the weight given to $I_{i}$

\section{$\mathrm{T}_{\mathrm{i}} \mathrm{x}_{\mathrm{i}}(\mathrm{t})$ is passive decay term}

If the weights are symmetric $\left(\mathrm{w}_{\mathrm{ij}}=\mathrm{w}_{\mathrm{ji}}\right)$ than it is a Hopfield model which is a feedback neural network called autoassociative memory. The hetro associative network is a network consisting of two layers of units and there exists weighted interconnection between the layers[9].The weights are $\mathrm{w}_{\mathrm{ij}}$ and $\mathrm{v}_{\mathrm{ij}}$ from the first layer to the second layer and vice versa [10][11]. If the weights are identical then it is a bidirectional model or bidirectional associative memory [12][13]. For the restriction of the range of activation values to a Specified range, Shunting activation model was proposed. The on-center off surround shunting activation model was introduced to make steady-state activation value sensitive to reflectance by providing inhibitory inputs from other input elements to the ith unit[3][13][14]. The shunting dynamics activation model is used to keep the operating range of activation value to a specified range. 


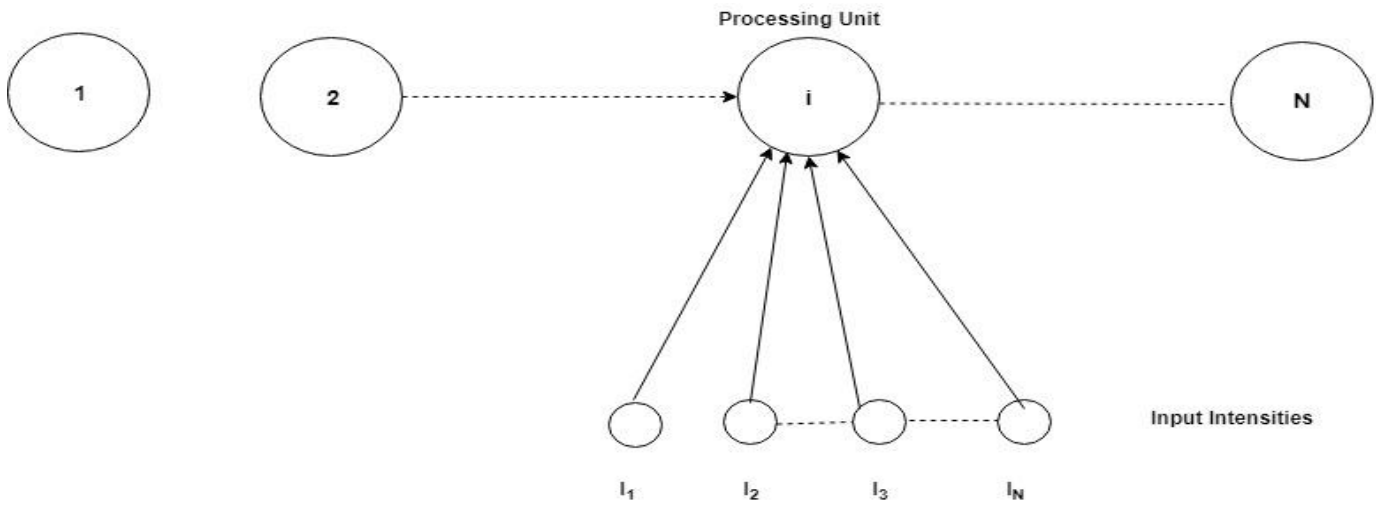

Figure 3: Shunting Activation Model (on Center and off-surround configuration)

Generally activation models are deterministic models. The activation value and input /output data are functions of random processes. The random function of activation value is the output generated. These are basically considered as vector stochastic processes [17] [18]. When additional noise component is added to the right side of expression for $x_{i}(t)$ are the Stochastic activation models. For analyzing the vector stochastic processes, the probability distribution of noise component is assumed.

\subsection{Synaptic Dynamics}

Synaptic dynamics define the weight vector as a function of time. For storing the patterns the role of weights takes place. The change in weights deals with Learning [17][18]. Learning is a process with the help of which a neural network adapts itself by making proper parameter adjustments, resulting in the making of the desired response. Learning may be based on deterministic methods ie Hebbian learning, Backpropagation or Stochastic methods ie genetic algorithms or simulated annealing. So the behavior of artificial neural network is the ability to learn, recollect and generalize training patterns or data just like human brain. For learning of pattern implicit, a large number of samples is required. The information of the patterns is spread across all the weights and it's complicate to relate weights to training samples. The method to guide the evidence of learning pattern is that given another sample from the same pattern source, the network would classify the new sample into the pattern class of earlier trained sample. The synaptic dynamics of network is described by the adjustment of synaptic weights is represented by learning equations. It is given by the expression for the first derivative of synaptic weights. The supervised learning helps in weight adjustment by determining the deviation of actual output to desired output. Temporal Learning can be used as supervised learning which helps in capturing the weights the relationship between neighboring patterns or Structural Learning can be used as supervised learning which helps in capturing weights the relationship between input and output pattern. Unsupervised learning uses local information that is activation values of the units at either end of the connection for weight update is to be made. The variables may be discrete or continuous in the implementation in learning and also the weights. So the network should learn from the training samples.

\subsection{Network Reliability}

This section presents a formal definition of the problem that how a highly reliable layered or the structured computer network can be designed so overall system can have a minimum fault and minimum cost. All-terminal reliability is the probability that a set of operational edges provides communication paths between every pair of nodes. A communications network is typically modeled as a graph with nodes, and edges. Nodes represent sites (computers), and Edges represent communication links. Each node and each edge has an associated probability of failure and the reliability of the network is the probability that the network is operational. With the following assumptions, we start our problem definition

a) Nodes are completely reliable; failure of links is the cause of network failure.

b) The probabilities of link failure are independent.

The problem is NP-hard as there exists no algorithm with a polynomial-time to compute all-terminal network reliability [15]. Although simulation is suitable for large networks it is more flexible than analytical methods, it has disadvantages. The most notable is that it gives approximations; and when more accuracy is necessary, the running time to provide the desired confidence interval can grow large. So we need to calculate the following

a) Is certain vertex is reachable from another given vertex that is to calculate the probability?

b) What is the probability of network overload?

c) Where are the feeble points of the network (e.g. w.r.t. security or reliability)?

d) Is a spread of failure within the network possible? How?

e) What is the probability of loss?

The exact evaluation of the reliability parameter is in the general case an NP-hard problem. The problem of maximizing reliability in highly increasing variable-size network is NP-hard, because the computational effort required to exactly find reliability is growing exponentially with the increase in a number of nodes and links in the networks. A computer or communication network can be modeled by a probabilistic $\operatorname{graph} G=(N, L, p)$, in which $N$ and $L$ represent set of network nodes and links in the network and $p$ is the link reliabilities for fixed and variable links. The network reliability measurement can be defined using following formula mentioned in as follows:

Minimize $C(X)$

and $R(X) \geq R_{O}$ 
$X=\left(X_{12}, \ldots, X_{i j}, . ., X_{N-1 N}\right) \geq R_{O}=\left(R_{O 1}, . ., R_{O 2}, \ldots, R_{O N}\right)$

The general objective is to design a computer by selecting a subset of possible links $X$ from highly increasing variable sized growing networks that layered networks of high reliability and minimum cost will be constructed. The objective is to find the minimum cost and fault tolerant layered architecture with high reliability is defined using Formula stated in as follows:

Minimize $Z(X)=\sum_{i=1}^{t} \sum_{j=i+1}^{n_{i}} C_{i j} X_{i j}$

And, reliability of each and every link and network node that communicates with every other node, to construct a highly reliable layered or structured network from highly increasing networks. Thus, reliability and cost will be two important considerations when efficient, effective and fault-tolerant network need from highly increasing network size. Reliability of each network node will be calculated and added to find uniform reliability of layered structure as follows:

$R(X)=\sum_{\Omega}\left[\prod_{j \in x^{\prime}} p\left(x_{j}\right)\right]\left[\prod_{j \in x / x^{\prime}} q\left(x_{j}\right)\right]$

Since, reliability is defined as probability that each and every network node communicate with every remaining node in the network. The reliable set of possible links will have probability is $p$ and $q=1-p$ is probability for nonreliable set of links, only some links of $X$ might be operational. A set of operational links of $X$ represented as a state $\left(N, X^{\prime}\right)$, where $X^{\prime}$ are specific operational links from $X$. Eq. (5) represents the state $X^{\prime} \subseteq X$ of the layered network structure, and $\Omega$ is a set of all operational states in the network summed together.

The state space size of possible topology will be formulated as

$K \times \frac{((\operatorname{Modof} N) \times(\operatorname{Modof} N)-1)}{2}$,

$K$ is the no. of possible links between any number of communicating nodes. This $K$ may be in two states i.e. either it is assumed that links may be connected or not i.e. $K \in(0,1)$ or random no. of links between nodes i.e. $K \in$ set of natural no.

\subsection{Reliability Measuring Approach}

1. Indicate the design parameters that are the number of input to ANN, the number of output signals, number of network layers in ANN, and also the number of hidden layers and learning rate.

2. Initial Values of parameters such as connection weights $(w)$, learning rate $(\alpha)$, number of trails to achieve epochs, and the values for tolerance are assigned.

3. The training of ANN done by using the BackPropagation training method.

4. This step is used to choose significant parameters such as network topology with high upper-bound for reliability, adaptive learning and convergence rate, and so on.
5. Based on the selection of a significant parameter, the neural network is reconfiguring.

6. If no significant parameters have been selected or specified number of epochs have been reached then stop the training.

\section{RESULTS}

The example of a graph is defined which is a Torus Network. The Torus network is more reliable for the given set of nodes and link reliability The Torus Network has the connection of opposite edges which gives more options to send data with high speed. The energy consumption is less as may use less number of hops. The Torus network for the given set of link reliability and nodes can tolerant more failures compared to the other networks.

Table 1: Probability of Link failure

\begin{tabular}{|c|r|r|r|r|}
\hline $\mathbf{P}(\mathbf{K})$ & $\mathbf{f 1}$ & $\mathbf{f 2}$ & $\mathbf{f 3}$ & $\mathbf{f 4}$ \\
\hline 2 & 0.9792 & 0.9812 & 0.9951 & 0.9981 \\
\hline 4 & 0.9901 & 0.9634 & 0.9951 & 0.9983 \\
\hline 6 & 0.9763 & 0.9447 & 0.9952 & 0.9963 \\
\hline 8 & 0.9764 & 0.9222 & 0.9953 & 0.9951 \\
\hline 10 & 0.9755 & 0.9342 & 0.9954 & 0.9941 \\
\hline 12 & 0.9746 & 0.9811 & 0.9955 & 0.9932 \\
\hline 14 & 0.9737 & 0.9612 & 0.9956 & 0.9927 \\
\hline
\end{tabular}

\section{Link failure Probability}

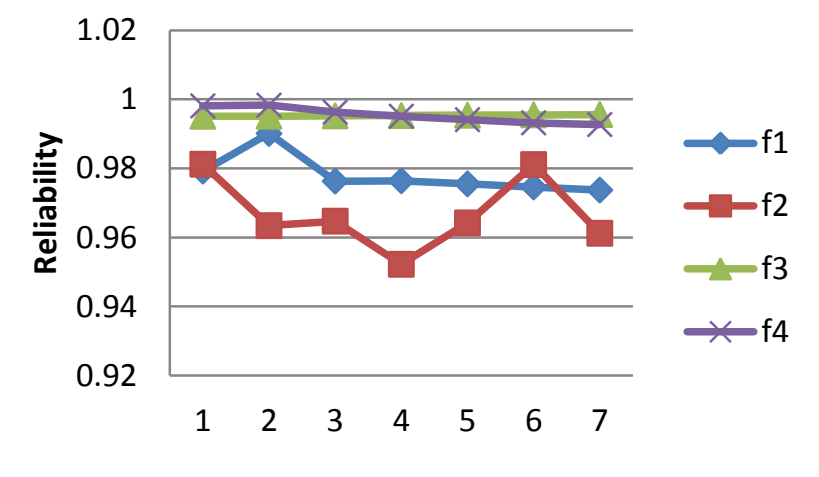

Figure 4: Probability of Link failure

Table 2: Existing Reliability and Estimated Reliability

\begin{tabular}{|c|c|c|c|c|}
\hline P(K) & P0 & R0 & $\begin{array}{c}\text { Existing } \\
\text { Reliability }\end{array}$ & Estimated \\
\cline { 4 - 5 } & & & & Reliability \\
\hline 2 & 0.843 & 0.941 & 0.9712 & 0.9107 \\
\hline 4 & 0.813 & 0.956 & 0.9656 & 0.9616 \\
\hline 6 & 0.812 & 0.934 & 0.9123 & 0.9231 \\
\hline 8 & 0.854 & 0.951 & 0.9561 & 0.9572 \\
\hline 10 & 0.832 & 0.932 & 0.9245 & 0.9255 \\
\hline 12 & 0.825 & 0.951 & 0.9099 & 0.9231 \\
\hline 14 & 0.829 & 0.952 & 0.9112 & 0.9123 \\
\hline
\end{tabular}




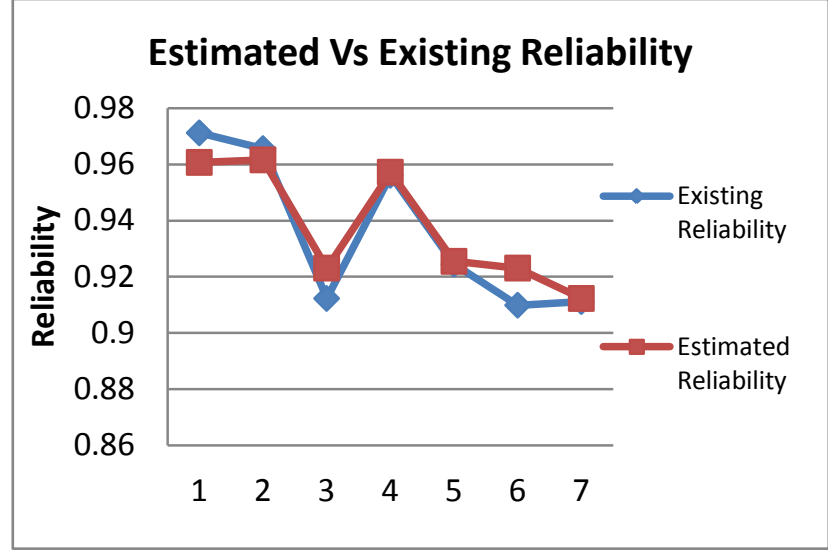

Figure 5: Estimated Vs Existing Reliability

The advantage of the Chordal network is the availability of multiple paths between source and destination of the same length. But as we increase the number of nodes the reliability drops and providing fault tolerance becomes a difficult task

\section{CONCLUSION}

This objective of this paper is to focus on the design of minimum cost reliable computer networks when a set of nodes, their topology, and links are given to connect them. Some of the basic concepts are introduced. The network design problem is difficult when the overall reliability measure is considered through the methods. For optimal network design, an optimized ANN is used. Using the proposed technique, it is possible to obtain a simple reliability evaluation expression for the general communication system. An ANN has several advantages but one of the most recognized of these is the fact that it. can actually learn from observing data sets. In this way, ANN is used as a random function approximation tool. Using the proposed technique, it is possible to obtain a simple reliability evaluation expression for the general communication system.

\section{REFERENCES}

[1]. D.E. Rumelhart, G.E. Hinton, and R.J. Williams, "Learning internal representation by error propagation," in Parallel Distributed Processing: Exploration in the Microstructure of Cognition. Cambridge,Massachusetts: MIT Press, 1986, vol. I, pp. 318-361.

[2]. Misha Tsodyks, Klaus Pawelzik, Henry Markram, "Neural Networks with Dynamic Synapses" Neural Computation, MIT 10, 821-835 (1998)

[3]. Abdullah Konak and Alice E "Designing Resilient Networks Using a Hybrid Genetic Algorithm Approach" GECCO'05, June 25-29, 2005, Washington, DC, USA. Copyright 2005 ACM 1-59593-010-8/05/0006

[4]. T. Elperin, I. Gertsbakh, M. Lomonosov, "An evolution model for Monte Carlo estimation of equilibrium network renewal parameters", Prob. Eng. Inf. Sci. 6(1992) 457-469.

[5]. Bibitchkov D., Herrmann J. M., Geisel T. (2002). Pattern storage and processing in attractor networks with shorttime synaptic dynamics. Netw. Comput. Neural Syst. 13, $115-129$
[6]. Y. Shpungin, "Combinatorial approaches to Monte Carlo estimation of dynamic systems reliability parameters," Comm. Depend. Qual. Manage. 9 (1) (2006)69-75.

[7]. Ramanathan, C.J. Colbourn, "Counting almost minimum cut sets with reliability" applications, Math. Program. 39 (2005) 253-261.

[8]. Anuradha and Anil Kumar "Calculation and Evaluation of Network Reliability using ANN Approach" International Conference on Computational Intelligence and Data Science (ICCIDS 2019), Northcap University, India

[9]. Zhongshi He, Yufang Tian, Yinong Chen,"Simulating the reliability of Distributed systems with unreliable nodes" ijssst.info/Vol-03/No-1\&2

[10]. Yegnanarayana . B, "Artificial Neural Network" 2009, 461p.ISBN 8120312538

[11].Bonifazi P., Ruaro M. E., Torre V. (2005). Statistical properties of information processing in neuronal networks. Eur. J. Neurosci. 22, 2953-2964

[12].Wang $\mathrm{XJ}(2008)$ Decision making in recurrent neuronal circuits. Neuron 60:215-234

[13].Mongillo G,Barak O,Tsodvks (2008) Synaptic theory of working memory Science319:1543-1546.

[14].Joaquin J. Torres and Hilbert J. Kappen "Emerging phenomena in neural networks with dynamic synapses and their computational implications" Front Comput Neurosci. 2013; 7: 30.

[15].Kilpatrick Z. P., Bressloff P. C. (2010). Spatially structured oscillations in a two-dimensional excitatory neuronal network with synaptic depression. J. Comput. Neurosci. 28, 193-209 10.1007/s10827-009-0199-6

[16]. Barak O., Tsodyks M. (2007). Persistent activity in neural networks with dynamic synapses. PLoS Comput. Biol 3:e35 10.1371/journal.pcbi.0030035

[17].Schalkoff Robert J., "Artificial Neural Network", 1997, 421p. ISBN 0-07-115554-6

[18].Mazzoni A., Broccard F. D., Garcia-Perez E., Bonifazi P., Ruaro M. E., Torre V. (2007). On the dynamics of the spontaneous activity in neuronal networks. PLoS ONE 2:e439 10.1371/journal.pone.0000439

[19].Collins J. J., Carson Chow C. C., Imhoff T. T. (1995). Aperiodic stochastic resonance in excitable systems. Phys. Rev. E 52, R3321-R3324 10.1103/PhysRevE.52.R3321

[20].Krahe R, Gabbiani F (2004) Burst firing in sensory systems. Nat Rev Neurosci 5: 13-23

[21].Duda Richard O. , Hart Peter E., Stork David G. "Pattern Classification" Wiley Student Edition ISBN: 978-81265-1116-7,654p.

[22]. Yasuda H., Miyaoka T., Horiguchi J., Yasuda A., Hanggi P., Yamamoto Y. (2008). Novel class of neural stochastic resonance and error-free information transfer. Phys. Rev. Lett. 100:118103 10.1103/PhysRevLett.100.118103 